\title{
Atypical Scar Patterns after Gastric Endoscopic Submucosal Dissection
}

\author{
Bomin Kim, Beom Jin Kim, Hong Jip Yoon, Hyunsuk Lee, Jae Yong Park, Chang Hwan Choi, Jae Gyu Kim \\ Department of Internal Medicine, Chung-Ang University Hospital, Chung-Ang University College of Medicine, Seoul, Korea
}

Background/Aims: Endoscopic submucosal dissection (ESD) for gastric neoplasms is a widely performed procedure. Local recurrence is rare, but various post-ESD scars are encountered during follow-up endoscopy. Therefore, we investigated atypical scar patterns and evaluated the associated factors.

Materials and Methods: Clinicopathologic and endoscopic reviews of gastric neoplasms treated with ESD from January 2009 to December 2015 were conducted. Atypical scar patterns were classified as irregular erythema, nodularity, or mucosal defect.

Results: A total of 264 patients with 274 gastric neoplasms, including 201 adenomas and 73 early gastric cancers, were enrolled. The key endoscopic findings at the resection scar were defined on the basis of gross morphology as follows: irregular erythema, mucosal defect (erosion or ulcer), and nodularity. An irregular erythema scar pattern was associated with male sex, a nodularity scar pattern with smoking, and a mucosal defect scar pattern with infra-angle location (angle and antrum) and cancer. An irregular erythema with nodularity scar pattern was also associated with male sex. An irregular erythema with nodularity and mucosal defect scar pattern was associated with liver disease and chronic kidney disease.

Conclusions: The atypical scar patterns after gastric ESD are associated with various clinicopathologic factors. (Korean J Helicobacter Up Gastrointest Res 2021;21:72-81)

Key Words: Endoscopic submucosal dissection; Scar; Stomach neoplasms

\section{INTRODUCTION}

Gastric cancer is a common malignancy and a major cause of morbidity and mortality worldwide. In East Asia, gastric cancer has shown high mortality rates. ${ }^{1}$ In recent years, deaths associated with gastric cancer have decreased markedly in some Asian countries, especially in Korea and Japan, owing to health-care policies and advances in diagnostic technology that lead to early detection of early gastric cancer (EGC) or premalignant lesions.

Surgical resection is the standard method of treatment; however, for the treatment of select patients with EGC, a paradigm shift away from surgery to endoscopic resection (ER) is increasingly being seen, because ER is beneficial for the treatment of selective EGC and even gastric adenoma, which is considered a premalignant lesion. ER has shown many advantages over surgical resection in terms

Received: August 30, 2020 Revised: October 25, 2020 Accepted: November 29, 2020

Corresponding author: Beom Jin Kim

Department of Internal Medicine, Chung-Ang University College of Medicine, 102 Heukseok-ro, Dongjak-gu, Seoul 06973, Korea

Tel: +82-2-6299-2060, Fax: +82-2-6299-2064, E-mail: kimbj@cau.ac.kr of less invasiveness, preservation of organ function, low cost, short hospital stay, and improvement of the quality of life. ${ }^{2}$ According to a recent study, endoscopic submucosal dissection (ESD) has demonstrated its effectiveness for the treatment of EGC or premalignant lesions in terms of high curative resection and low adverse event rates. ${ }^{3,4}$ ESD with specialized devices is the most advanced and preferred technique for the treatment of gastric tumors. This technique, which allows en bloc resection of larger or ulcerated lesions, reduces the recurrence rate as compared with endoscopic mucosal resection. $^{2,5-7}$ Therefore, ESD is now accepted as the standard ER technique for EGC and premalignant lesions. ${ }^{8}$ Despite the success of ESD, however, the healing process of iatrogenic ulcers should also be considered in postprocedural management. The risk factors for local recurrence after ER for gastric neoplasms are known to be incomplete resection (e.g., piecemeal resection), undifferentiated histology, tumor-positive resection margin, lymphovascular invasion, and invasion depth greater than one-third of the submucosa. ${ }^{6,9}$ Local recurrence is rare but various post-ESD scars are encountered during fol-

Copyright $\odot 2021$ Korean College of Helicobacter and Upper Gastrointestinal Research

@ The Korean Journal of Helicobacter and Upper Gastrointestinal Research is an Open-Access Journal. All articles are distributed under the terms of the Creative Commons Attribution Non-Commercial License (http:// creativecommons.org/licenses/by-nc/4.0) which permits unrestricted non-commercial use, distribution, and reproduction in any medium, provided the original work is properly cited. 
low-up endoscopy. However, which endoscopic findings are suggestive of local recurrence remains unclear; the necessity of routinely taking a biopsy at the resection scar has also not been determined.

To date, there have been few studies focusing on the relationship between risk factors for tumor recurrence and an iatrogenic ulcer scar appearance. ${ }^{10,11}$ Indeed, during follow-up endoscopy, a lesion that is not confirmed as a local recurrence on biopsy may have a morphology suspicious for recurrence. This situation causes confusion in the diagnosis of local recurrence. Especially in patients with a bleeding tendency, unnecessary biopsy may result in fatal consequences. Therefore, we investigated the atypical scar patterns and evaluated the factors associated with such a condition.

\section{MATERIALS AND METHODS}

\section{Patients and study design}

From January 2009 to December 2015, patients who underwent ESD for gastric neoplasms at Chung-Ang University Hospital (Seoul, Korea) were initially enrolled. Gastric adenoma and carcinoma, especially EGC, were categorized as gastric neoplasms in this study. Gastric neoplasm was confirmed with forceps biopsy under esophagogastroduodenoscopy (EGD) guidance (GIF-H260 or GIF-Q260; Olympus, Tokyo, Japan). During EGD, if dysplasia or carcinoma was suspected, two to four biopsy samples were obtained with forceps (FB-25 K-1 [Olympus] or BX 420 [PriMed Instruments
Inc., Ontario, Canada]). ESD was indicated when the following criteria were met: any lesions with low- to high-grade dysplasia, regardless of size, or well- to moderately differentiated adenocarcinoma confined to the mucosa without evidence of lymph node or distant metastases on abdominal computed tomography or endoscopic ultrasonography. However, in some patients, the pathological results after ESD were revealed to be outside of the criteria. In such cases, whether to perform additional surgery or ESD was decided according to the physician's discretion and the patient's wish. The following patients were excluded from the study: those who had no endoscopy at the 3-month follow-up visit and those who underwent surgical resection after endoscopic treatment owing to adverse events or lymphovascular or margin invasion in the pathological specimen. The medical records of patients were reviewed with regard to age, sex, medication, Helicobacter pylori (H. pylori) infection, and endoscopic characteristics including tumor size, location, macroscopic type, and gross morphology. The study protocol was approved by the Institutional Review Board (IRB) of Chung-Ang University Hospital, Korea (IRB no. C2015155).

\section{Evaluation of endoscopic characteristics}

Each endoscopic finding and report at 3 months follow-up were reviewed to determine the size, location, macroscopic type, and gross morphology of the lesion. The location of lesions was defined according to seven sections of the stomach: antrum, angle, lower body, mid body, high body, fundus, and cardia. The location of the
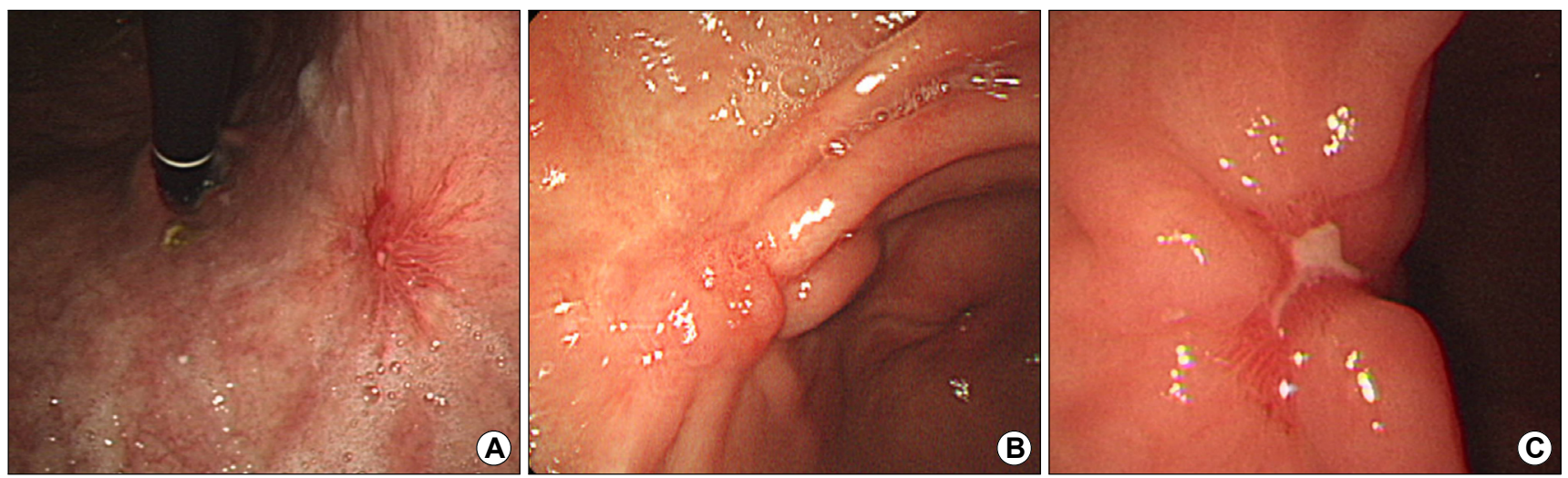

Fig. 1. Endoscopic classification relating to the endoscopic resection scar. (A) Irregular erythema. (B) Nodularity. (C) Mucosal defect. 
lesion was classified following the Japanese Classification of Gastric Carcinoma. ${ }^{12}$ According to the Paris endoscopic classification, the macroscopic type of the lesion was divided into the superficial elevated, flat, or depressed types. ${ }^{13}$ In addition, gross morphology including erythema, ulcer, erosion, or nodularity, $H$. pylori infection, intestinal metaplasia, and chronic atrophic gastritis were also examined.

In the present study, the key endoscopic findings at the resection scar were defined on the basis of gross morphology, as follows: irregular erythema, mucosal defect (erosion or ulcer), and nodularity (Fig. 1). We defined irregular erythema at the resection scar as an irregular reddish mucosal change, and we also included findings of engorged capillary vessels at the scar into irregular erythema. Ulcer was defined as a $\geq 5$-mm mucosal break with a well-defined crater, whereas erosion was defined as a superficial mucosal defect without an ulcer crater. ${ }^{14}$ Nodularity was defined as the presence of an irregularly raised or nodular surface without a dominant mass. ${ }^{15}$ In this study, recurrent tumor at the resection site after 12 months was defined as local recurrence.

\section{Procedure}

All lesions were removed through ESD by three experienced gastrointestinal endoscopists (B.J.K., J.G.K., and C.H.C) by using a single-channel endoscope (GIF-Q260J, GIF-H260Z, or GIF-H290; Olympus). The patients were sedated by using a course of intravenous midazolam (0.05 $\mathrm{mg} / \mathrm{kg}$ ) or propofol and remifentanil, with a target-controlled infusion system under bispectral index (BIS) monitoring by an experienced anesthesiologist. The initial target effect-site concentration of propofol and remifentanil was $1.5 \mu \mathrm{g} / \mathrm{mL}$ and $1.5 \mathrm{ng} / \mathrm{mL}$, respectively. The plasma concentration was increased or decreased by $0.2 \mu \mathrm{g} / \mathrm{mL}$ or $0.2 \mathrm{ng} / \mathrm{mL}$, targeting a 60 80 BIS score.

In all patients, dot markings for ESD were first placed $5 \mathrm{~mm}$ beyond the tumor margins with argon plasma

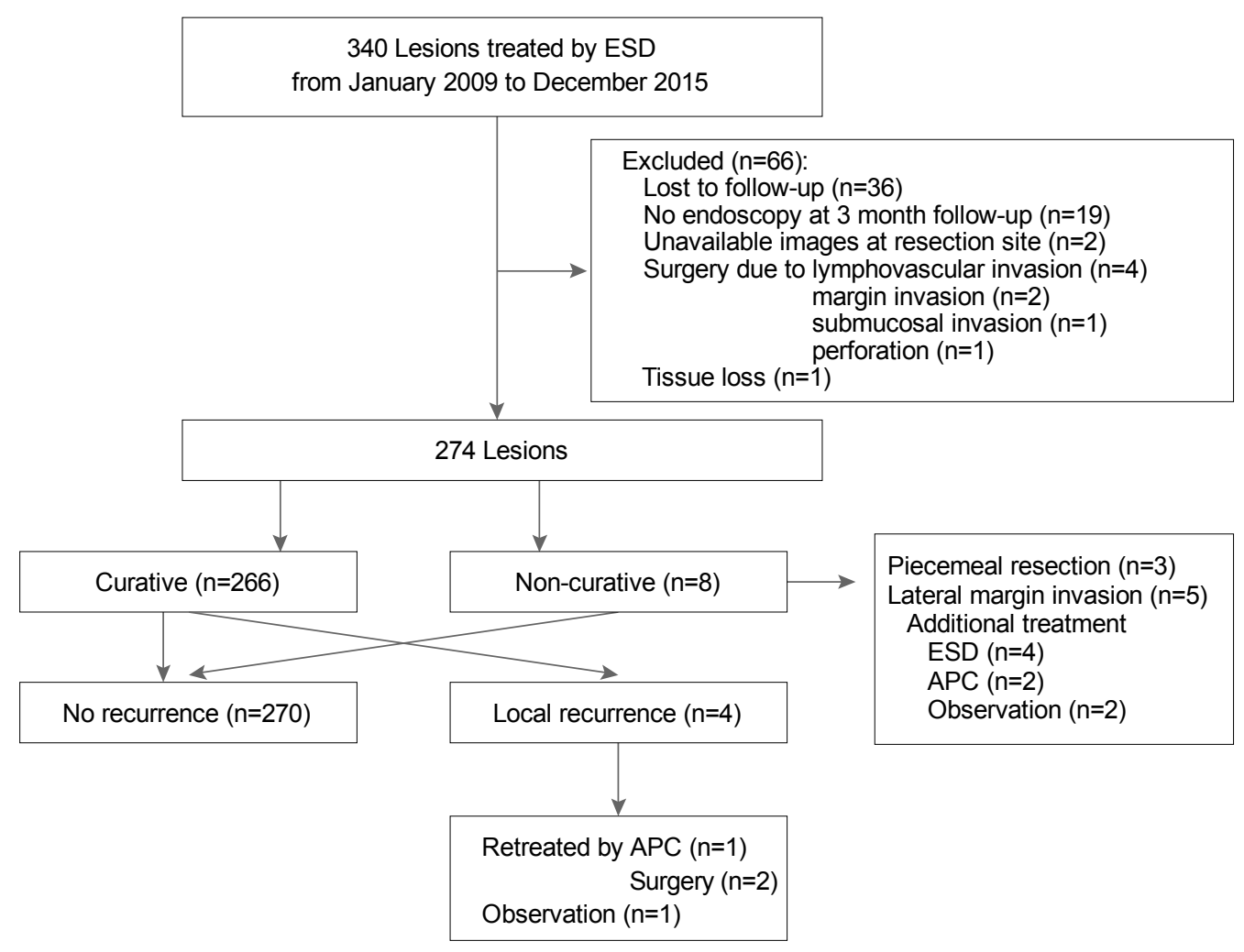

Fig. 2. Clinical outcomes of 274 gastric tumors treated by endoscopic submucosal dissection. ESD, endoscopic submucosal dissection; APC, argon plasma coagulation. 
coagulation. A saline solution (100 $\mathrm{mL} 0.9 \%$ saline mixed with $1 \mathrm{mg}$ epinephrine and a small amount of indigo carmine) was then injected into the submucosal layer, and the submucosal layer around the lesion was dissected by using a needle knife (Nadel-Papillotom, 99020121; MTW Endoskopie, Wesel, Germany), insulated-tip knife (KD-611L; Olympus), and/or flex knife (KD-630L; Olympus). If necessary, saline injection was repeated and endoscopic hemostasis was achieved during the procedure. A high-frequency electrosurgical current generator (VIO 300D; ErbeElektromedizin, Tübingen, Germany) was used during the processes of marking, mucosal incision, submucosal dissection, and hemostasis. A complete resection was defined as a resection in which the resected tumor had tumor-free horizontal and vertical margins. Complete en bloc resection was defined as en bloc resection achieved endoscopically along with histological confirmation of tumor-free margins.

After ESD, all patients received $40 \mathrm{mg}$ pantoprazole intravenously or took $30 \mathrm{mg}$ lansoprazole orally once daily on the day they underwent ESD and throughout the length of their hospital stay, which was usually $4 \sim 5$ days. If no ESD-related adverse events occurred, the patients were allowed a liquid diet on the first or second day after ESD. The patients were discharged the day after starting diet. After discharge, the patients were instructed to take $30 \mathrm{mg}$ lansoprazole once a day for 4 weeks. The patients were examined with conventional endoscopy at 3, 6, and 12 months after ER and annually thereafter.

\section{Evaluation of pathologic specimens}

At our center, pathologic diagnosis was made on the basis of the third edition of the Japanese Classification of Gastric Carcinoma. ${ }^{11}$ The pathologic type, depth of invasion, lymphatic and venous involvement, and the presence of tumor at the resection margin (horizontal and vertical) were evaluated.

\section{Statistical analysis}

Statistical analysis was performed with SPSS ver. 21.0 (Statistical Package for Social Sciences; SPSS Inc., Chicago, IL, USA). For univariate analysis, categorical variables were analyzed by using a chi-square test or Fisher exact test. Those variables with $P<0.20$ in the univariate analyses were examined in multivariate analysis by using binary logistic regression models. A $P$ value of $<0.05$ was considered significant.

Table 1. Baseline Clinicopathologic Characteristics of 274 Lesions in 264 Patients

\begin{tabular}{|c|c|}
\hline & Value \\
\hline Age (years) & $64.4 \pm 9.3$ \\
\hline \multicolumn{2}{|l|}{ Sex } \\
\hline Male & $175(66.3)$ \\
\hline Female & $89(33.7)$ \\
\hline \multicolumn{2}{|l|}{ Underlying diseases } \\
\hline Hypertension & $106(40.2)$ \\
\hline Diabetes mellitus & $46(17.4)$ \\
\hline Chronic kidney disease & $11(4.2)$ \\
\hline Liver cirrhosis & $5(1.9)$ \\
\hline Cardiovascular disease & $19(7.2)$ \\
\hline Smoking & $83(31.4)$ \\
\hline Alcohol drinking & $124(47.0)$ \\
\hline History of peptic ulcer & $3(1.1)$ \\
\hline \multicolumn{2}{|l|}{ Medication } \\
\hline Aspirin & $37(14.0)$ \\
\hline Antiplatelet agent & $15(5.7)$ \\
\hline NSAIDs & $7(2.7)$ \\
\hline Anticoagulant & $1(0.4)$ \\
\hline Steroid & $1(0.4)$ \\
\hline \multicolumn{2}{|l|}{ Location } \\
\hline Antrum/angle & $248(90.5)$ \\
\hline Body/fundus/cardia & $26(9.5)$ \\
\hline \multicolumn{2}{|l|}{ Macroscopic type } \\
\hline Superficial elevated & $246(89.8)$ \\
\hline Flat or superficial depressed & $28(10.2)$ \\
\hline \multicolumn{2}{|l|}{ Lesion size } \\
\hline$<2 \mathrm{~cm}$ & $208(75.9)$ \\
\hline$\geq 2 \mathrm{~cm}$ & $66(24.1)$ \\
\hline Helicobacter pylori infection & $82(29.9)$ \\
\hline \multicolumn{2}{|l|}{ Histopathology } \\
\hline Adenoma & $201(73.4)$ \\
\hline Mucoal cancer & $70(25.5)$ \\
\hline Submucosal cancer & $3(1.1)$ \\
\hline
\end{tabular}

Values are presented as mean \pm standard deviation or number (\%). NSAID, non-steroidal anti-inflammatory drug. 


\section{RESULTS}

A total of 301 gastric neoplasms were removed by ESD at Chung-Ang University Hospital between January 2009 and December 2015. Among them, 16 were excluded because of follow-up loss, two because of unavailable images at the resection site, eight because of an additional operation, and one because of tissue loss after ER. Therefore, the remaining 274 neoplasms, including 201 adenomas and 73 EGCs, were

Table 2. Therapeutic Outcomes from Endoscopic Submucosal Dissection of 274 Lesions

\begin{tabular}{lc}
\hline & Value \\
\hline Procedure time (minutes) & $56.3 \pm 35.0$ \\
En bloc resection & $271(98.9)$ \\
Yes & $3(1.1)$ \\
No & \\
Complete resection & $266(97.0)$ \\
Yes & $8(3.0)$ \\
No & \\
Curative resection & $266(97.0)$ \\
Yes & $8(3.0)$ \\
No & \\
Adverse events & $0(0.0)$ \\
Significant bleeding & $3(1.1)$ \\
Perforation & $1(0.4)$ \\
Delayed bleeding & \\
Scar pattern & 101 \\
Irregular erythema & 85 \\
Nodularity & 104 \\
Mucosal defect & $24.6 \pm 19.3$ \\
Follow-up duration (months) & $4(1.5)$ \\
Recurrence &
\end{tabular}

Values are presented as mean \pm standard deviation or number (\%). finally enrolled (Fig. 2).

\section{Baseline clinicopathologic characteristics}

The baseline clinicopathologic characteristics of the study population are summarized in Table 1 . The mean age of the patients was 64.4 years, and $66.3 \%$ of them were men. Comorbidities including hypertension ( $n=106)$, diabetes mellitus $(n=46)$, cardiovascular disease $(n=19)$, chronic kidney disease $(n=11)$, and liver cirrhosis $(n=5)$ were found. Nine patients had a history of peptic ulcer. Eighty-three patients were current smokers, whereas 124 patients were alcohol drinkers. The number of patients who were taking aspirin was 37, and 15 patients were taking an antiplatelet agent other than aspirin, seven were taking nonsteroidal anti-inflammatory drugs, and one was taking an anticoagulant and steroid.

Among the total lesions resected by using ESD, 201 were adenomas and 73 were EGCs. Almost lesions were located at the antrum or angle, and the macroscopic shape of most lesions was superficially elevated. The mean size of the tumor was $14.6 \mathrm{~mm}$, and the mean area of the tumor was $212.4 \mathrm{~mm}^{2}$. The H. pylori infection was observed in 82 patients.

\section{Therapeutic outcomes of endoscopic submucosal dissection}

Therapeutic outcomes are summarized in Table 2. En bloc resection rate was 98.9\% (271/274). Complete resection rate and curative resection rate were 97.0\% (266/274) and 97.0\% (266/274), respectively. With regard to the gross morphol-

Table 3. Clinicopathologic Characteristics of Patients with Tumor Recurrence

\begin{tabular}{|c|c|c|c|c|c|c|c|c|c|c|c|c|c|}
\hline $\begin{array}{l}\text { Patient } \\
\text { No. }\end{array}$ & $\begin{array}{c}\text { Age } \\
\text { (years) }\end{array}$ & Sex & Diagnosis & $\begin{array}{l}\text { Comorbi } \\
\text {-dity }\end{array}$ & Smoking & $\begin{array}{l}\text { Tumor } \\
\text { location }\end{array}$ & $\begin{array}{l}\text { Specimen } \\
\text { size }(\mathrm{mm})\end{array}$ & $\begin{array}{l}\text { Curative } \\
\text { resection }\end{array}$ & $\begin{array}{c}\text { Follow-up } \\
\text { duration } \\
\text { (months) }\end{array}$ & $\begin{array}{l}\text { Irregular } \\
\text { erythema }\end{array}$ & Nodularity & $\begin{array}{c}\text { Mucosal } \\
\text { defect }\end{array}$ & $\begin{array}{l}\text { Additional } \\
\text { treatment }\end{array}$ \\
\hline 1 & 64 & $\mathrm{~F}$ & LGA & HT, DM & No & Antrum & 26 & Yes & 76 & No & Yes & No & Surgery \\
\hline 2 & 78 & M & Cancer & $\mathrm{DM}, \mathrm{CKD}$ & Yes & Antrum & 26 & Yes & 17 & Yes & Yes & Yes & $\begin{array}{l}\text { Observa } \\
\text {-tion }\end{array}$ \\
\hline 3 & 66 & M & LGA & No & No & Antrum & 25 & Yes & 35 & Yes & No & No & APC \\
\hline 4 & 70 & $\mathrm{M}$ & LGA & LC & Yes & Cardia & 31 & Yes & 19 & Yes & No & Yes & Surgery \\
\hline
\end{tabular}

LGA, low-grade adenoma; HT, hypertension; DM, diabetes mellitus; CKD, chronic kidney disease; APC, Argon plasma coagulation; LC, liver cirrhosis. 
ogy of the scar, 101 lesions (36.9\%) were classified as irregular erythema, 85 (31.0\%) as nodular lesions, and 104 (38.0\%) as unhealed mucosal defects. The mean follow-up duration was 24.5 months. There were four recurrence cases (Table 3). Concerning additional treatment methods, two patients with recurrence underwent surgical resection, whereas one underwent argon plasma coagulation treatment. The remaining one patient had low-grade dysplasia and refused to undergo further treatment, although endoscopic follow-up had been done.

\section{Factors associated with atypical scar patterns}

Atypical scar patterns were defined as irregular erythema, nodularity, or mucosal defect. In univariate analysis for the irregular erythema scar pattern, the following factors showed marginal significance $(P<0.100)$ : male sex and specimen size $>40 \mathrm{~mm}$ (Table 4). Likewise, the nod-

Table 4. Univariate Analysis of Scar Patterns for Predicting Tumor Recurrence

\begin{tabular}{|c|c|c|c|c|c|c|c|c|c|c|c|c|c|c|c|}
\hline & \multicolumn{3}{|c|}{ Irregular erythema } & \multicolumn{3}{|c|}{ Nodularity } & \multicolumn{3}{|c|}{ Mucosal defect } & \multicolumn{3}{|c|}{$\begin{array}{c}\text { Irregular } \\
\text { erythema+nodularity }\end{array}$} & \multicolumn{3}{|c|}{$\begin{array}{c}\text { Irregular } \\
\text { erythema+nodularity+ } \\
\text { mucosal defect }\end{array}$} \\
\hline & OR & $95 \% \mathrm{CI}$ & $P$-value & OR & $95 \% \mathrm{CI}$ & $P$-value & OR & $95 \% \mathrm{CI}$ & $P$-value & OR & $95 \% \mathrm{CI}$ & $P$-value & OR & $95 \% \mathrm{CI}$ & $P$-value \\
\hline Male & 2.30 & $1.32 \sim 4.02$ & 0.003 & 2.24 & $1.24 \sim 4.03$ & 0.007 & 0.95 & $0.57 \sim 1.60$ & 0.854 & 3.92 & $1.48 \sim 10.42$ & 0.003 & 6.54 & $0.84 \sim 50.00$ & 0.067 \\
\hline Age $\geq 65$ years & 0.81 & $0.49 \sim 1.32$ & 0.392 & 0.60 & $0.36 \sim 1.00$ & 0.050 & 1.37 & $0.83 \sim 2.24$ & 0.217 & 0.67 & $0.34 \sim 1.34$ & 0.257 & 0.67 & $0.22 \sim 2.03$ & 0.471 \\
\hline $\begin{array}{l}\text { Alcohol } \\
\text { drinking }\end{array}$ & 1.45 & $0.89 \sim 2.38$ & 0.136 & 1.64 & $0.98 \sim 2.75$ & 0.058 & 0.63 & $0.38 \sim 1.03$ & 0.063 & 1.57 & $0.79 \sim 3.12$ & 0.198 & 1.41 & $0.46 \sim 4.32$ & 0.542 \\
\hline Smoking & 1.46 & $0.87 \sim 2.47$ & 0.153 & 3.22 & $1.87 \sim 5.54$ & $<0.001$ & 0.52 & $0.30 \sim 0.91$ & 0.020 & 2.22 & $1.11 \sim 4.46$ & 0.022 & 0.97 & $0.29 \sim 3.24$ & 1.000 \\
\hline HT & 1.25 & $0.76 \sim 2.06$ & 0.378 & 0.92 & $0.55 \sim 1.56$ & 0.765 & 1.02 & $0.62 \sim 1.67$ & 0.950 & 1.41 & $0.71 \sim 2.80$ & 0.328 & 1.29 & $0.42 \sim 3.96$ & 0.651 \\
\hline $\mathrm{DM}$ & 1.28 & $0.68 \sim 2.41$ & 0.447 & 1.01 & $0.52 \sim 1.98$ & 0.970 & 0.98 & $0.51 \sim 1.86$ & 0.943 & 1.31 & $0.56 \sim 3.06$ & 0.537 & 1.44 & $0.38 \sim 5.44$ & 0.706 \\
\hline Liver disease & 1.15 & $0.19 \sim 6.97$ & 1.000 & 9.28 & $1.02 \sim 84.35$ & 0.033 & 1.09 & $0.18 \sim 6.64$ & 1.000 & 4.32 & $0.70 \sim 26.72$ & 0.143 & 15.64 & $2.37 \sim 103.32$ & 0.019 \\
\hline CVD & 1.44 & $0.58 \sim 3.61$ & 0.433 & 1.22 & $0.47 \sim 3.16$ & 0.690 & 0.68 & $0.25 \sim 1.83$ & 0.446 & 1.62 & $0.51 \sim 5.13$ & 0.497 & 2.46 & $0.51 \sim 11.93$ & 0.243 \\
\hline CKD & 1.45 & $0.43 \sim 4.88$ & 0.541 & 1.28 & $0.37 \sim 4.51$ & 0.743 & 2.02 & $0.60 \sim 6.80$ & 0.247 & 2.44 & $0.62 \sim 9.65$ & 0.184 & 5.09 & $0.98 \sim 26.42$ & 0.032 \\
\hline $\begin{array}{l}\text { History of } \\
\text { PUD }\end{array}$ & 0.34 & $0.04 \sim 2.92$ & 0.419 & 0.44 & $0.05 \sim 3.81$ & 0.669 & 0.61 & $0.56 \sim 0.67$ & 0.086 & 0.34 & $0.04 \sim 2.92$ & 0.419 & 0.95 & $0.93 \sim 0.98$ & 1.000 \\
\hline Hazard drug & 1.14 & $0.62 \sim 2.08$ & 0.673 & 0.86 & $0.45 \sim 1.65$ & 0.657 & 0.98 & $0.53 \sim 1.79$ & 0.937 & 1.25 & $0.55 \sim 2.81$ & 0.593 & 1.18 & $0.31 \sim 4.43$ & 0.733 \\
\hline HP infection & 0.78 & $0.45 \sim 1.35$ & 0.378 & 1.23 & $0.71 \sim 2.13$ & 0.465 & 1.53 & $0.91 \sim 2.60$ & 0.110 & 0.81 & $0.38 \sim 1.76$ & 0.600 & 1.49 & $0.47 \sim 4.71$ & 0.539 \\
\hline $\begin{array}{l}\text { Location } \\
\text { (infra-angle) }\end{array}$ & 0.65 & $0.29 \sim 1.47$ & 0.302 & 0.70 & $0.30 \sim 1.60$ & 0.389 & 8.40 & $1.94 \sim 35.71$ & $<0.001$ & 0.39 & $0.15 \sim 1.00$ & 0.043 & 1.06 & $1.03 \sim 1.09$ & 0.620 \\
\hline Metaplasia & 0.83 & $0.50 \sim 1.38$ & 0.472 & 1.03 & $0.60 \sim 1.74$ & 0.928 & 0.96 & $0.58 \sim 1.59$ & 0.864 & 1.14 & $0.56 \sim 2.35$ & 0.715 & 0.93 & $0.30 \sim 2.93$ & 1.000 \\
\hline Cancer & 1.38 & $0.80 \sim 2.39$ & 0.247 & 0.86 & $0.48 \sim 1.56$ & 0.627 & 3.04 & $1.75 \sim 5.28$ & $<0.001$ & 1.14 & $0.54 \sim 2.44$ & 0.729 & 1.77 & $0.56 \sim 5.61$ & 0.341 \\
\hline $\begin{array}{l}\text { Tumor } \\
\text { morphology } \\
\text { (flat or } \\
\text { depressed) }\end{array}$ & 0.95 & $0.42 \sim 2.14$ & 0.894 & 1.06 & $0.46 \sim 2.45$ & 0.892 & 0.75 & $0.33 \sim 1.73$ & 0.503 & 1.40 & $0.50 \sim 3.95$ & 0.562 & 2.83 & $0.73 \sim 10.97$ & 0.136 \\
\hline $\begin{array}{l}\text { Lesion size } \\
\geq 20 \mathrm{~mm}\end{array}$ & 1.48 & $0.84 \sim 2.60$ & 0.171 & 1.05 & $0.58 \sim 1.91$ & 0.872 & 1.64 & $0.94 \sim 2.87$ & 0.083 & 1.80 & $0.86 \sim 3.75$ & 0.116 & 0.56 & $1.12 \sim 2.59$ & 0.740 \\
\hline $\begin{array}{l}\text { Specimen size } \\
\geq 40 \mathrm{~mm}\end{array}$ & 1.63 & $0.91 \sim 2.90$ & 0.097 & 0.74 & $0.39 \sim 1.41$ & 0.359 & 1.98 & $1.11 \sim 3.53$ & 0.019 & 0.92 & $0.40 \sim 2.13$ & 0.847 & 0.28 & $0.04 \sim 2.19$ & 0.309 \\
\hline $\begin{array}{l}\text { Hemostasis } \\
\text { at SLE }\end{array}$ & 1.18 & $0.65 \sim 2.17$ & 0.589 & 1.22 & $0.65 \sim 2.29$ & 0.527 & 1.48 & $0.81 \sim 2.69$ & 0.200 & 1.52 & $0.69 \sim 3.35$ & 0.301 & 2.64 & $0.83 \sim 8.41$ & 0.090 \\
\hline $\begin{array}{l}\text { Procedural } \\
\text { duration } \\
\geq 1 \text { hour }\end{array}$ & 1.51 & $0.91 \sim 2.50$ & 0.110 & 1.66 & $0.98 \sim 2.80$ & 0.058 & 0.88 & $0.53 \sim 1.46$ & 0.613 & 2.17 & $1.09 \sim 4.33$ & 0.026 & 2.11 & $0.69 \sim 6.46$ & 0.183 \\
\hline
\end{tabular}

OR, odds ratio; CI, confidence interval; HT, hypertension; DM, diabetes mellitus; CVD, cardiovascular disease; CKD, chronic kidney disease; PUD, peptic ulcer disease; HP, Helicobacter pylori; SLE, second-look endoscopy. 
ularity scar pattern was related to male sex, age younger than 65 years, alcohol drinking, smoking, and a procedural duration $>1$ hour. Mucosal defect was related to alcohol drinking, smoking (inverse relationship), history of peptic ulcer disease, infra-angle location (angle and antrum), cancer, lesion size $>20 \mathrm{~mm}$, and specimen size $>40$ $\mathrm{mm}$. The irregular erythema with nodularity scar pattern was related to male sex, smoking, infra-angle location, and procedural duration $>1$ hour. Furthermore, the irregular erythema with nodularity and mucosal defect scar pattern was related to male sex, liver disease, chronic kidney disease, and hemostasis at second-look EGD in univariate analysis. These related factors were examined with multivariate analysis. Table 5 showed that male sex was an independent risk factor of the irregular erythema scar pattern (OR, 2.25; 95\% CI, 1.15-4.39; $P=0.018$ ) and smoking was an independent risk factor of the nodularity scar pattern (OR, 2.51; 95\% CI, 1.30-4.85; $P=0.006$ ). In addition, infra-angle location (OR, 8.20; 95\% CI, 1.74-38.4; $P=0.008$ ) and cancer were related to the $m u-$ cosal defect scar pattern as independent risk factors (OR, 2.81; 95\% CI, 1.54-5.14; $P=0.001$ ). Male sex was demonstrated as an independent risk factor of the irregular erythema with nodularity scar pattern (OR, 3.46; 95\% CI,

Table 5. Multivariate Analysis of Scar Patterns for Predicting Tumor Recurrence

\begin{tabular}{|c|c|c|c|c|c|c|c|c|c|c|c|c|c|c|c|}
\hline & \multicolumn{3}{|c|}{ Irregular erythema } & \multicolumn{3}{|c|}{ Nodularity } & \multicolumn{3}{|c|}{ Mucosal defect } & \multicolumn{3}{|c|}{$\begin{array}{c}\text { Irregular } \\
\text { erythema+nodularity }\end{array}$} & \multicolumn{3}{|c|}{$\begin{array}{c}\text { Irregular } \\
\text { erythema+nodularity+ } \\
\text { mucosal defect }\end{array}$} \\
\hline & OR & $95 \% \mathrm{CI}$ & $P$-value & OR & $95 \% \mathrm{CI}$ & $P$-value & OR & $95 \% \mathrm{CI}$ & $P$-value & OR & $95 \% \mathrm{CI}$ & $P$-value & OR & $95 \% \mathrm{CI}$ & $P$-value \\
\hline Male & 2.25 & $1.15 \sim 4.39$ & 0.018 & 1.48 & $0.72 \sim 1.59$ & 0.288 & 1.63 & $0.82 \sim 3.25$ & 0.164 & 3.46 & $1.13 \sim 10.64$ & 0.030 & 5.18 & $0.64 \sim 41.67$ & 0.124 \\
\hline $\begin{array}{c}\text { Age } \geq 65 \\
\text { years }\end{array}$ & 0.83 & $0.49 \sim 1.40$ & 0.480 & 0.76 & $0.43 \sim 1.34$ & 0.342 & 1.26 & $0.72 \sim 2.22$ & 0.424 & 0.62 & $0.28 \sim 1.38$ & 0.245 & 0.71 & $0.19 \sim 2.64$ & 0.604 \\
\hline $\begin{array}{l}\text { Alcohol } \\
\text { drinking }\end{array}$ & 1.03 & $0.56 \sim 1.90$ & 0.924 & 0.89 & $0.46 \sim 1.71$ & 0.719 & 0.71 & $0.36 \sim 1.41$ & 0.333 & 0.95 & $0.41 \sim 2.19$ & 0.896 & & & \\
\hline Smoking & 0.99 & $0.53 \sim 1.87$ & 0.983 & 2.51 & $1.30 \sim 4.85$ & 0.006 & 0.50 & $0.25 \sim 1.01$ & 0.054 & 1.21 & $0.52 \sim 2.80$ & 0.665 & & & \\
\hline Liver disease & & & & 7.17 & $0.73 \sim 70.10$ & 0.090 & & & & 4.80 & $0.68 \sim 33.71$ & 0.115 & 16.99 & $1.72 \sim 167.88$ & 0.015 \\
\hline $\begin{array}{l}\text { History of } \\
\text { PUD }\end{array}$ & & & & & & & 0.00 & 0.00 & 0.999 & & & & & & \\
\hline CKD & & & & & & & & & & 3.91 & $0.86 \sim 17.77$ & 0.078 & 12.71 & $1.79 \sim 90.27$ & 0.011 \\
\hline HP infection & & & & & & & 1.66 & $0.93 \sim 2.96$ & 0.088 & & & & & & \\
\hline $\begin{array}{l}\text { Location } \\
\text { (infra-an- } \\
\text { gle) }\end{array}$ & & & & & & & 8.20 & $1.74 \sim 38.46$ & 0.008 & 0.39 & $0.14 \sim 1.10$ & 0.075 & & & 0.138 \\
\hline Cancer & & & & & & & 2.81 & $1.54 \sim 5.14$ & 0.001 & & & & & & \\
\hline $\begin{array}{l}\text { Tumor mor- } \\
\text { phology } \\
\text { (flat or de- } \\
\text { pressed) }\end{array}$ & & & & & & & & & & & & & 1.60 & $0.32 \sim 8.54$ & 0.564 \\
\hline $\begin{array}{l}\text { Lesion size } \\
\geq 20 \mathrm{~mm}\end{array}$ & 1.29 & $0.66 \sim 2.51$ & 0.454 & & & & 1.63 & $0.82 \sim 3.25$ & 0.165 & 1.66 & $0.71 \sim 3.88$ & 0.246 & & & \\
\hline $\begin{array}{l}\text { Specimen } \\
\text { size } \geq 40 \\
\mathrm{~mm}\end{array}$ & 1.34 & $0.69 \sim 2.60$ & 0.394 & & & & 1.05 & $0.52 \sim 2.13$ & 0.886 & & & & & & \\
\hline $\begin{array}{l}\text { Hemostasis } \\
\text { at SLE }\end{array}$ & & & & & & & & & & & & & 3.13 & $0.81 \sim 12.06$ & 0.098 \\
\hline $\begin{array}{l}\text { Procedural } \\
\text { duration } \\
\geq 1 \text { hour }\end{array}$ & 1.32 & $0.75 \sim 2.33$ & 0.333 & 1.56 & $0.89 \sim 2.72$ & 0.118 & & & & 1.93 & $0.87 \sim 4.28$ & 0.106 & 2.42 & $0.69 \sim 8.54$ & 0.168 \\
\hline
\end{tabular}

OR, odds ratio; CI, confidence interval; PUD, peptic ulcer disease; CKD, chronic kidney disease; HP, Helicobacter pylori; SLE, second-look endoscopy. 
1.13-10.64; $P=0.030$ ). Liver disease and chronic kidney disease were demonstrated as independent risk factors of the irregular erythema with nodularity and mucosal defect scar pattern (OR, 16.99; 95\% CI, 1.72-167.88; $P=0.015$ and $\mathrm{OR}, 12.71 ; 95 \% \mathrm{CI}, 1.79-90.27 ; P=0.011$, respectively).

\section{DISCUSSION}

Although follow-up endoscopy usually detects a hypertrophied and/or hyperemic scar at the ER site, it is not known whether these findings are suggestive of local recurrence. A recent study reported that several typical endoscopic findings such as irregular erythema, nodularity, mucosal defect, and a combination of these patterns might predict the local recurrence after ESD for EGCs. ${ }^{10}$ In the present study, we defined these findings as atypical scar patterns, especially suggesting local recurrence. As a result, we demonstrated that these atypical scar shapes were significantly related to some clinicopathologic factors such as male sex, infra-angle location, histopathology, liver disease, and chronic kidney disease. To our knowledge, this is the first study to analyze post-ESD scars and their association with various clinicopathologic factors.

In the early days since ER had been introduced, concerns were focused on the outcomes of curative resection rate and technical feasibility. However, as large experience accumulates, ESD is currently being applied to a wide variety of indications, with sufficient curability. Therefore, the issue of local recurrence or metachronous lesions is receiving more attention recently. Moreover, the healing process of post-ER ulcers is still not completely understood.

In general, after a successful curative ER, follow-up endoscopy is expected to demonstrate a homogeneous and flat epithelized scar covered by normal mucosa with some grade of fibrosis. ${ }^{11}$ However, not all post-ER scars have a regular or homogenous mucosal pattern. Therefore, some studies sought to predict local recurrence at the ER scar on the basis of gross morphologic criteria without biopsy. $\mathrm{Na}$ et al. ${ }^{10}$ reported that one or a combination of features such as an unevenly superficial elevated morphology, hyperemic change, and mucosal defect in the post-ER scar might be related to local recurrence. For example, an unevenly super- ficial elevated morphology with hyperemic change had shown high specificity (94.3\%), negative predictive value (99.6\%), and accuracy (86.4\%). In addition, an unevenly superficial elevated morphology with hyperemic change and mucosal defect had also shown high specificity (100\%), positive predictive value (100\%), negative predictive value (99.8\%), and accuracy (86.4\%). Interestingly, all patients with local recurrence showed more than one of these scar patterns in the present study.

Irregular erythema and irregular erythema with nodular scar change were significantly more frequently noted in the male group in multivariate analysis. In another study, gastric ulcer or gastric ulcer perforation was predominant in male patients. ${ }^{16}$ It is possible that delayed healing of ulcers in men might have affected irregular erythema or irregular erythema with nodular scar formation.

Nodular scar change after ER was significantly related to smoking in multivariate analysis. A number of studies have provided evidence that smoking is a major cause of gastrointestinal disorders, including chronic inflammation such as peptic ulcer and inflammatory bowel disease and cancer of the gastrointestinal tract. ${ }^{17-19}$ Especially, according to clinical observation, smokers are more likely to develop ulcers that are more difficult to heal. ${ }^{20}$ The risk of peptic ulcer also increases in smokers who have a large daily intake of tobacco compared with never smokers. ${ }^{21}$ This increased risk may be due to the adverse effects of smoking related to the reduction of antioxidants or the defensive immune system locally present in the gastroduodenal mucosa. Perhaps these adverse effects of smoking affect the healing of post-ER scars, as they seem to interfere with the smooth healing of the scar.

Mucosal defect was related to the histopathology (cancer) and location of the lesion (angle and antrum) in multivariate analysis. Tumor location might be as influential in the healing process for ER-induced ulcers. ${ }^{22}$ Furthermore, previous reports have shown that postoperative bleeding occurred more frequently in the lower third than in the upper or middle third of the stomach. ${ }^{23,24}$ Owing to a bleeding tendency in the lower third of the stomach, electrocoagulation might be performed in this location more frequently than in other locations. Electrocoagulation can deliver thermal energy deep into the proper muscle, unlike argon plasma 
coagulation. Therefore, hemostasis with electrocoagulation may interrupt the natural healing process by inducing fibrosis in the muscle layer that is critical for wound contraction in the early phase, and ablate the supplying blood vessels underneath the ulcer floor. Therefore, the ER scar in the lower third of the stomach might not heal well.

The irregular erythema with nodularity and mucosal defect scar pattern was significantly related to liver disease and chronic kidney disease. Although the pathophysiology of this phenomenon is incompletely understood, the incidence of, frequency of bleeding from, and severity of bleeding from peptic ulcer disease are all increased in patients with liver disease. ${ }^{25}$ Defensive mucosal factors, including gastric mucosal perfusion and mucosal secretion, are impaired in the presence of portal hypertension. ${ }^{26}$ In long-term follow-up studies, patients with chronic kidney disease were found to be at an increased risk for peptic ulcer disease than the general population. ${ }^{27,28}$ Furthermore, these data are relevant with regard to higher rates of peptic ulcer bleeding complications and mortality compared with the general population. ${ }^{29}$ In chronic kidney disease, metabolic alterations of uremia favor bacterial overgrowth in the gut and increase the translocation of living bacteria and bacterial components, and this may affect peptic ulcer. Therefore, that peptic ulcer and its complications occur frequently in patients with liver disease and chronic kidney disease is likely to be associated with irregular and delayed ulcer healing.

This study has some limitations. First, there may have been a potential selection or information bias resulting from the retrospective nature of the study. Second, our study had a relatively small number of patients. Therefore, a further large-scale prospective study involving a greater number of patients is warranted.

In conclusion, this study revealed the clinicopathologic factors related to atypical post-ESD scars, especially suspicious for local recurrence. Although local recurrence is rare after ESD, screening for local recurrence is important. However, the risk of bleeding may increase if excessive biopsy is performed in patients with a bleeding tendency (patients with chronic kidney disease or liver disease, those taking antiplatelets or anticoagulation agents, etc.). Therefore, understanding the factors associated with atypical post-ESD scar morphology may reduce the bleeding risk from unnecessary biopsy.

\section{CONFLICTS OF INTEREST}

No potential conflict of interest relevant to this article was reported.

\section{ORCID}

$\begin{array}{ll}\text { Bomin Kim } & \text { (D) https://orcid.org/0000-0001-8547-1335 } \\ \text { Beom Jin Kim } & \text { (D https://orcid.org/0000-0002-0938-6697 } \\ \text { Hong Jip Yoon } & \text { (D https://orcid.org/0000-0002-0859-7644 } \\ \text { Hyunsuk Lee } & \text { (D https://orcid.org/0000-0002-3160-7222 } \\ \text { Jae Yong Park } & \text { (D) https://orcid.org/0000-0001-6114-8920 } \\ \text { Chang Hwan Choi (D https://orcid.org/0000-0001-7089-532X } \\ \begin{array}{ll}\text { Jae Gyu Kim } & \text { (D) https://orcid.org/0000-0002-4841-9404 }\end{array}\end{array}$

\section{REFERENCES}

1. Ferlay J, Shin HR, Bray F, Forman D, Mathers C, Parkin DM. Estimates of worldwide burden of cancer in 2008: GLOBOCAN 2008. Int J Cancer 2010;127:2893-2917.

2. Soetikno R, Kaltenbach T, Yeh R, Gotoda T. Endoscopic mucosal resection for early cancers of the upper gastrointestinal tract. J Clin Oncol 2005;23:4490-4498.

3. Park YM, Cho E, Kang HY, Kim JM. The effectiveness and safety of endoscopic submucosal dissection compared with endoscopic mucosal resection for early gastric cancer: a systematic review and metaanalysis. Surg Endosc 2011;25:2666-2677.

4. Yamamoto H. Endoscopic submucosal dissection--current success and future directions. Nat Rev Gastroenterol Hepatol 2012;9:519-529.

5. Oka S, Tanaka S, Kaneko I, et al. Endoscopic submucosal dissection for residual/local recurrence of early gastric cancer after endoscopic mucosal resection. Endoscopy 2006;38: 9961000.

6. Takenaka R, Kawahara Y, Okada H, et al. Risk factors associated with local recurrence of early gastric cancers after endoscopic submucosal dissection. Gastrointest Endosc 2008;68:887-894.

7. Gotoda T. Endoscopic resection of early gastric cancer. Gastric Cancer 2007;10:1-11.

8. Park CH, Yang DH, Kim JW, et al. Clinical practice guideline for endoscopic resection of early gastrointestinal cancer. Korean J Gastroenterol 2020;75:264-291.

9. Jang JS, Choi SR, Qureshi W, et al. Long-term outcomes of endoscopic submucosal dissection in gastric neoplastic lesions at a single institution in South Korea. Scand J Gastroenterol 
2009;44:1315-1322.

10. Na HK, Choi KD, Ahn JY, et al. Endoscopic prediction of recurrence in patients with early gastric cancer after margin-negative endoscopic resection. J Gastroenterol Hepatol 2016;31:1284-1290.

11. Arantes V, Uedo N, Pedrosa MS, Tomita Y. Clinical relevance of aberrant polypoid nodule scar after endoscopic submucosal dissection. World J Gastrointest Endosc 2016;8:628-634.

12. Japanese Gastric Cancer Association. Japanese classification of gastric carcinoma: 3rd English edition. Gastric Cancer 2011; 14:101-112.

13. The Paris endoscopic classification of superficial neoplastic lesions: esophagus, stomach, and colon: November 30 to December 1, 2002. Gastrointest Endosc 2003;58(6 Suppl):S3-S43.

14. Ng TM, Fock KM, Khor JL, et al. Non-steroidal anti-inflammatory drugs, Helicobacter pylori and bleeding gastric ulcer. Aliment Pharmacol Ther 2000;14:203-209.

15. Choi J, Kim SG, Im JP, Kim JS, Jung HC, Song IS. Comparison of endoscopic ultrasonography and conventional endoscopy for prediction of depth of tumor invasion in early gastric cancer. Endoscopy 2010;42:705-713.

16. Li CH, Chang WH, Shih SC, Lin SC, Bair MJ. Perforated peptic ulcer in southeastern Taiwan. J Gastroenterol Hepatol 2010;25: 1530-1536.

17. WHO urges more countries to require large, graphic health warnings on tobacco packaging: the WHO report on the global tobacco epidemic, 2011 examines anti-tobacco mass-media campaigns. Cent Eur J Public Health 2011;19:133-151.

18. Chu KM, Cho CH, Shin VY. Nicotine and gastrointestinal disorders: its role in ulceration and cancer development. Curr Pharm Des 2013;19:5-10.

19. Zhang L, Ren JW, Wong CC, et al. Effects of cigarette smoke and its active components on ulcer formation and healing in the gastrointestinal mucosa. Curr Med Chem 2012;19:63-69.
20. Parasher G, Eastwood GL. Smoking and peptic ulcer in the Helicobacter pylori era. Eur J Gastroenterol Hepatol 2000;12: 843-853.

21. Andersen IB, Jørgensen T, Bonnevie O, Grønbaek M, Sørensen TI. Smoking and alcohol intake as risk factors for bleeding and perforated peptic ulcers: a population-based cohort study. Epidemiology 2000;11:434-439.

22. Yoshizawa Y, Sugimoto M, Sato Y, et al. Factors associated with healing of artificial ulcer after endoscopic submucosal dissection with reference to Helicobacter pylori infection, CYP2C19 genotype, and tumor location: multicenter randomized trial. Dig Endosc 2016;28:162-172.

23. Okano A, Hajiro K, Takakuwa H, Nishio A, Matsushita M. Predictors of bleeding after endoscopic mucosal resection of gastric tumors. Gastrointest Endosc 2003;57:687-690.

24. Takizawa K, Oda I, Gotoda T, et al. Routine coagulation of visible vessels may prevent delayed bleeding after endoscopic submucosal dissection--an analysis of risk factors. Endoscopy 2008;40:179-183.

25. Nojkov B, Cappell MS. Distinctive aspects of peptic ulcer disease, Dieulafoy's lesion, and Mallory-Weiss syndrome in patients with advanced alcoholic liver disease or cirrhosis. World J Gastroenterol 2016;22:446-466.

26. Kitano S, Dolgor B. Does portal hypertension contribute to the pathogenesis of gastric ulcer associated with liver cirrhosis? J Gastroenterol 2000;35:79-86.

27. Garrow D, Delegge MH. Risk factors for gastrointestinal ulcer disease in the US population. Dig Dis Sci 2010;55:66-72.

28. Liang CC, Muo CH, Wang IK, et al. Peptic ulcer disease risk in chronic kidney disease: ten-year incidence, ulcer location, and ulcerogenic effect of medications. PLoS One 2014;9:e87952.

29. Kang JY, Ho KY, Yeoh KG, et al. Peptic ulcer and gastritis in uraemia, with particular reference to the effect of Helicobacter pylori infection. J Gastroenterol Hepatol 1999;14:771-778. 